\title{
Analysis of $\mathrm{SiO}_{2}$ Thin Films Deposited by PECVD Using an Oxygen-TEOS-Argon Mixture
}

\author{
Carlos E. Viana, Ana N. R. da Silva, Nilton I. Morimoto, \\ LSI - EPUSP, São Paulo, SP, Brazil \\ and Olivier Bonnaud \\ GMV, UPRESA 6076, University of Rennes I, Rennes, France
}

Received on 15 May, 2000

\begin{abstract}
This study analyses the influence of the argon flow on the Plasma Enhanced Chemical Vapor Deposition (PECVD) of silicon oxide thin films by using TEOS as silicon source. The argon flow increases the deposition rate, however it also can creates some defects in the deposited film. Several characterization techniques were used to analyze the deposited films. The presence of argon, in the gas phase, modifies the plasma composition, the surface roughness of silicon wafer, and the surface reaction. The optimum argon flow ranges between 65 and $80 \mathrm{sccm}$ to obtain a silicon oxide thin film with high quality in terms of refractive index, smoothness, and uniformity.
\end{abstract}

\section{Introduction}

Plasma Enhanced Chemical Vapor Deposition (PECVD) by using tetraethylorthosilicate (TEOS), as silicon source, is a well-known technique to deposit silicon oxide thin films $[1,2]$. The advantage of this technique is to deposit silicon oxide with a high rate and at low temperature, that is compatible with a large range of applications. Indeed, as well in very large integrated circuit process with very thin junctions as in large area electronics using glass substrates, a temperature lower than $600^{\circ} \mathrm{C}$ is required [3]. However, for this rather low temperature, the silicon oxide film can present structural and electrical defects. Thus, additional studies are needed to optimize the process to improve the material. A way to modify the film properties is to change the dissociation conditions of TEOS and thus to add a gas in the plasma. We propose, in this work, to use a mixture of argon, oxygen and TEOS in the plasma [4]. Then, we analyze the influence of the argon flow on the silicon oxide thin films deposited by PECVD onto silicon substrate. The presence of argon modifies the plasma composition and the deposition rate and also can create some defects, more especially at the $\mathrm{Si} / \mathrm{SiO}_{2}$ interface. The main objective is thus to optimize the quality of the silicon oxide film by changing the argon flow.

After a presentation of the experimental conditions of the developed process, several explanations of the observed phenomena are given on the base of physical analyses and optimal conditions are drawn.

\section{Experimental}

The home made PECVD cluster tool system, used in this study, was described before $[5,6]$. It has three process chambers, a load lock and a sample manipulation chamber. Silicon wafers (100), $p$-type, 10-20 $\Omega . c m$, $75 \mathrm{~mm}$ in diameter were used as substrate. The substrates were cleaned using the piranha and RCA standard cleaning processes followed by a dip in diluted HF. The silicon oxide depositions were carried out in the conditions presented in Table 1. The TEOS, oxygen, and argon gases are mixed in a special chamber before entering the process reactor to guarantee a homogeneous mixture. The argon flow, expressed in sccm, is the analyzed parameter.

From Ellipsometry measurements, using a wavelength of $632.8 \mathrm{~nm}$, the thickness and the refractive index were determined. For the wet etching rate measurement, we used a diluted HF solution (1:100).

The Composition of the plasma, in term of molecules and radicals, is deduced from Optical Emission Spectroscopy (OES). Fourier Transform Infrared Spectroscopy (FTIRS) was used to determine the chemical bonding states of the films. To evaluate the contamination as well at the $\mathrm{Si} / \mathrm{SiO}_{2}$ interface as in the volume of the films, Atomic Force Microscopy (AFM) and $4 \mu$-RAMAN techniques were performed. 
Table 1: process parameters used in all the silicon oxide deposition processes.

\begin{tabular}{lc}
\hline \hline TEOS flow $(\mathrm{sccm})$ & 6.5 \\
Oxygen flow $(\mathrm{sccm})$ & 450 \\
Argon flow $(\mathrm{sccm})$ & 0 to 200 \\
Process pressure (Torr) & 1 \\
Temperature $\left({ }^{\circ} \mathrm{C}\right)$ & 375 \\
RF power $(\mathrm{W})$ & 400 \\
Distance between electrodes $(\mathrm{mm})$ & 10 \\
\hline
\end{tabular}

After the deposition process, some samples were annealed in a conventional furnace at $600^{\circ} \mathrm{C}$ during 12 hours, aluminum contact MOS capacitors were implemented for I-V measurements. The aluminum layer was annealed at $430^{\circ} \mathrm{C}$ in forming gas during $30 \mathrm{~min}$. The final capacitor test cell area is $300 \times 300 \mu \mathrm{m}^{2}$.

\section{Results and Discussions}

The influence of argon flow on the deposition and etching rates were analyzed.

Fig. 1 shows the deposition and etching rate in function of the argon flow. One can observe two regions. The first one (0-65 sccm of argon) in which the deposition rate decreases, the second one (100-200 sccm of argon) in which the deposition rate increases. In the first region, the slight decreasing of the etching rate shows that the density of the deposited films increases. This can be attributed to the argon bombardment of the surface, which desorbs the active species. In the gas phase, the effect of the argon is not significant, despite its higher atomic mass of the argon ion compared with the oxygen, because the partial pressure is too low. In the second region, the surface bombardment always increases but the deposition rate increases, which is attributed to the higher collision rate in the gas phase promoting the decomposition of the TEOS $[7,8]$. But it provokes also structural defects as confirmed by AFM and $\mu$-Raman results.

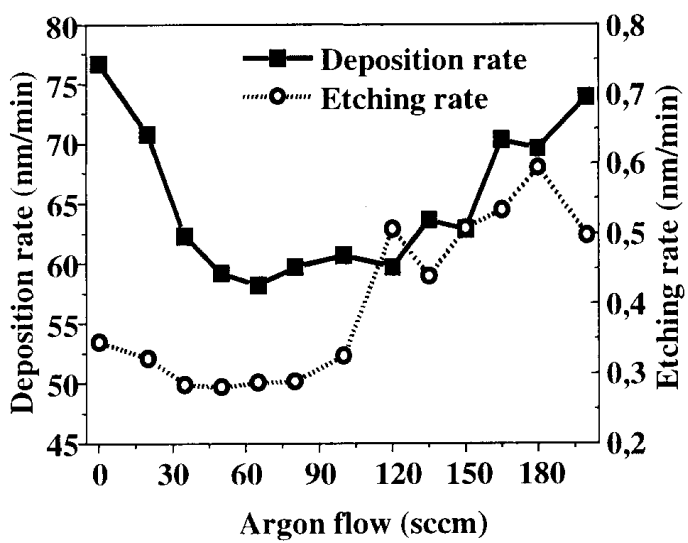

Figure 1. Deposition and etching rates of the silicon oxide films as a function of the argon flow. An increasing argon flow induces first a decrease and then an increase of the deposition rate with a correlated modification of the etching rate.
The refractive index of the silicon oxide films was $1.458 \pm 0.007$ and thus close to thermal oxide one. Moreover, we observed that the refractive index had no significant variation in depth, during the wet etching experiment. This means that despite the variation of the etching rate, the composition of the film is almost constant and the porosity remains low.

\section{1) OES analysis}

To determine the composition of the plasma and to understand the role of argon flow, OES analysis was performed $[7,8]$. Fig. 2 shows the oxygen, carbon monoxide, and argon optical emission intensities in function of argon flow.

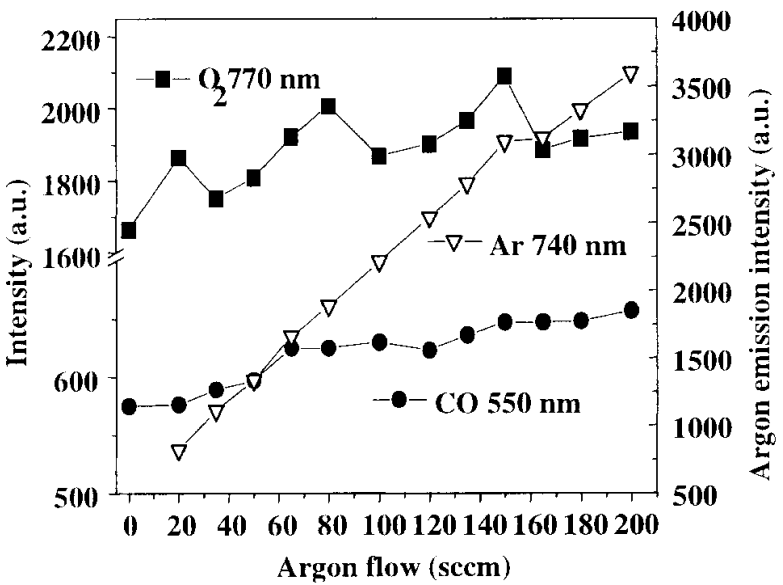

Figure 2. OES measurements of the $\mathrm{O}, \mathrm{CO}$ and Ar emission intensity in the gas phase during deposition. The $\mathrm{CO}$ emission increases with the argon flow suggesting an enhancement of the TEOS oxidation.

The slight and constant increase of the observed CO emission suggests an enhancement of the TEOS oxidation process by the argon added to the gas mixture. An increase of $\mathrm{O}_{2}$ emission is also observed that means that the argon enhances the generation of excited oxygen in the plasma.

\section{2) FTIRS analysis}

Fig. 3 shows a typical spectra of the deposited silicon oxide. For the argon flow (0-65 sccm), we observed the regular stretching, bending and rocking absorbance bands of silicon oxide films and also the $\mathrm{Si}-\mathrm{OH}$ absorbance band. For higher argon flow (100-200 sccm) the $\mathrm{Si}-\mathrm{OH}$ absorbance band was not detected showing low hydrogen incorporation during the deposition $[9,10,11]$. Moreover, the independence of the spectra, in function of the argon flow, confirms the almost constant composition as mentioned above despite the variations of the deposition and etching rates. 


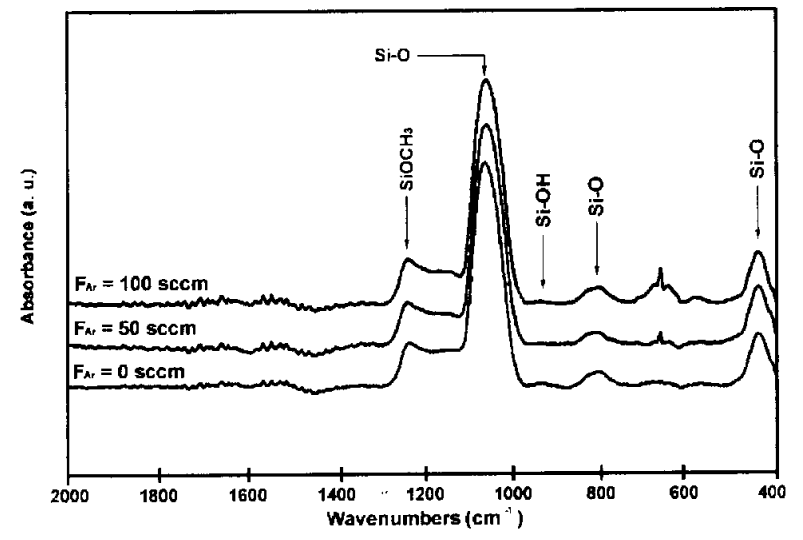

Figure 3. FTIRS spectra of samples deposited with different argon flows added to the total flux. The three observed peaks correspond to silicon-oxygen bonds; no $\mathrm{Si}-\mathrm{OH}$ peaks are present.

\section{3) AFM analysis}

Fig. 4 shows the AFM images of the silicon wafer surface after removing the silicon oxide layer.

First of all, an increase of the argon flow, up to $100 \mathrm{sccm}$, leads to an increase of the average roughness (see Table 2). This can be explained by the increasing bombardment of the silicon surface at the beginning of the deposition process, by the more numerous species present in the plasma. As mentioned above, this bombardment always increases with the argon flow.

For argon flows higher than $100 \mathrm{sccm}$, we observe a strong decrease of the roughness. This behavior can be explained by the breaking of the adsorbed TEOS subproducts at the silicon surface by the higher bombardment of argon ions. This bombardment also decreases the initial silicon roughness.

Moreover, one can observe the presence of small particles at the surface, which were not removed by the HF solution treatment. These particles are the unreacted $\left[(\mathrm{Et}-\mathrm{O})_{3} \mathrm{Si}-\mathrm{OH}\right]$ radicals remaining onto the silicon surface. As well for the lowest as for highest argon flows, the particle density is higher. This can be correlated to the deposition rate. When the deposition rate increases, these adsorbed particles have not reacted with oxygen and some of them remain on the surface and are not removed by the HF treatment. The effect of the plasma bombardment, for high argon flows, promotes an increasing of the roughness and defects density of the silicon surface increasing the probability of trapping of the unreacted TEOS radicals. This result is confirmed by $\mu$-Raman analysis.

Table 2: AFM measurements obtained from the samples showed in Fig. 4.

\begin{tabular}{cccc}
\hline \hline $\begin{array}{c}\text { Argon } \\
\text { Flow } \\
(\mathrm{sccm})\end{array}$ & $\begin{array}{c}\text { Particles/Area } \\
\text { Density } \\
\left(\mu \mathrm{m}^{-2}\right)\end{array}$ & $\begin{array}{c}\text { RMS } \\
\text { Roughness } \\
(\mathrm{nm})\end{array}$ & $\begin{array}{c}\text { Peak-Valley } \\
\text { Roughness } \\
(\mathrm{nm})\end{array}$ \\
\hline 0 & 88 & 0.54 & 47 \\
50 & 35 & 0.47 & 42 \\
100 & 13 & 0.61 & 77 \\
120 & 33 & 0.05 & 0.03 \\
135 & 44 & 0.12 & 0.14 \\
150 & 44 & 0.06 & 0.04 \\
168 & 41 & 0.12 & 0.21 \\
180 & 41 & 0.12 & 0.30 \\
200 & 39 & 0.09 & 0.12 \\
\hline \hline
\end{tabular}

\section{4. $\mu$-RAMAN analysis}

Punctual and large defects in the silicon oxide thin films are observed on $\mu$-RAMAN images as shown in Fig. 5.

The so-called "wafer defect" in this figure is related to the pre-existing line defect on the wafer surface. We expected that the so-called punctual defects have the same origin. Fig. 6 shows the $\mu$-RAMAN spectrum made inside the punctual defect. The presence of $\mathrm{C}=\mathrm{C}$ bonds confirms that the unreacted radicals are trapped by the surface structural defects.

This means that the "punctual defects" are carbonbased defects and come from unreacted TEOS fragments adsorbed at the surface at the beginning of the deposition process. Note that the wafer defects just reveal this phenomenon. One can note that except at these localized disturbances, the film is uniform and smooth showing an average good quality. 


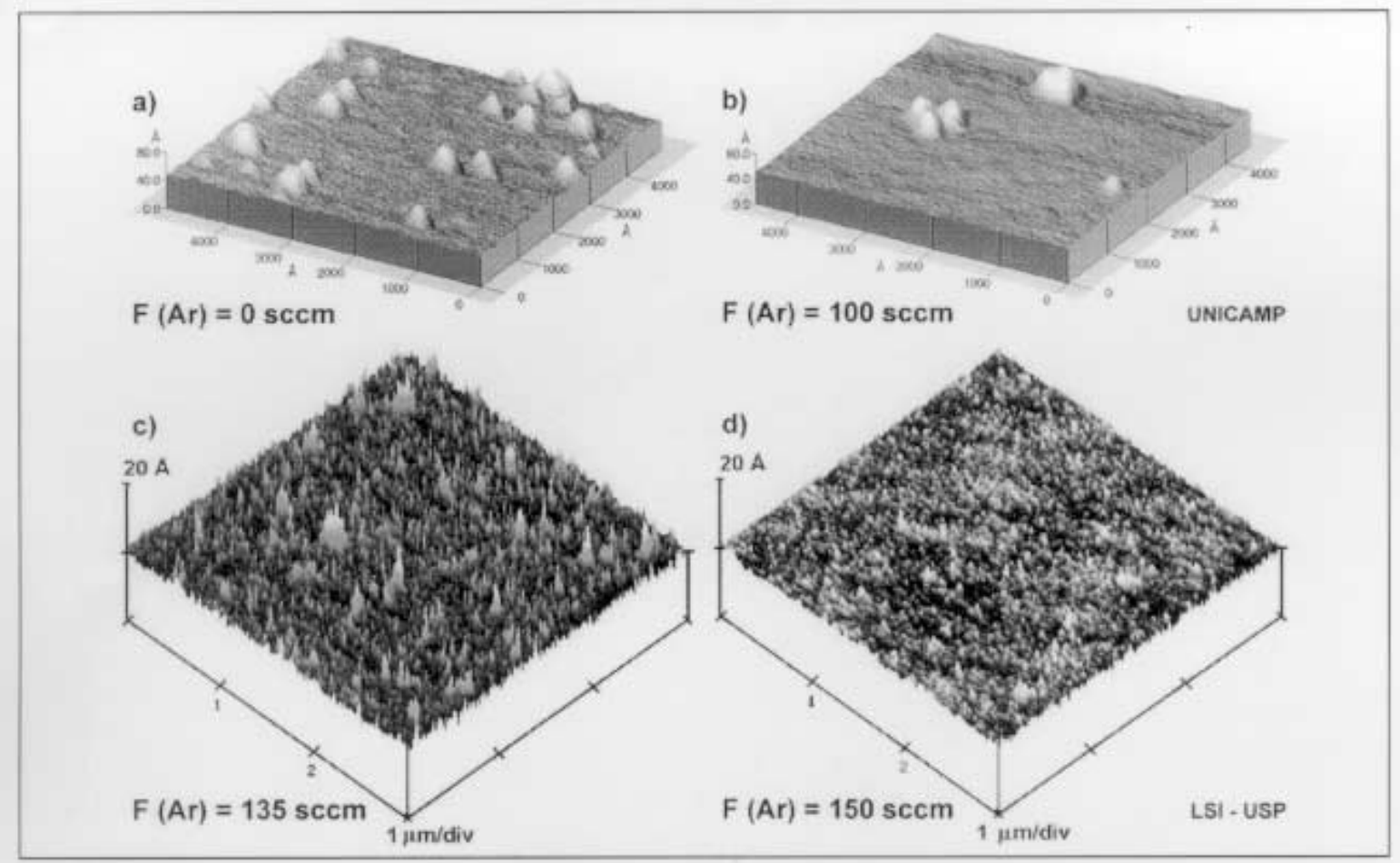

Figure 4. AFM images of the silicon wafer surface after removing the silicon oxide layer (using a diluted $\mathrm{HF}$ 1:100 $\mathrm{H}_{2} \mathrm{O}-\mathrm{DI}$ solution) and according to the argon flow variation: a) without the argon flow, b) $100 \mathrm{sccm}, \mathrm{c}) 135 \mathrm{sccm}$ and d) $150 \mathrm{sccm}$.

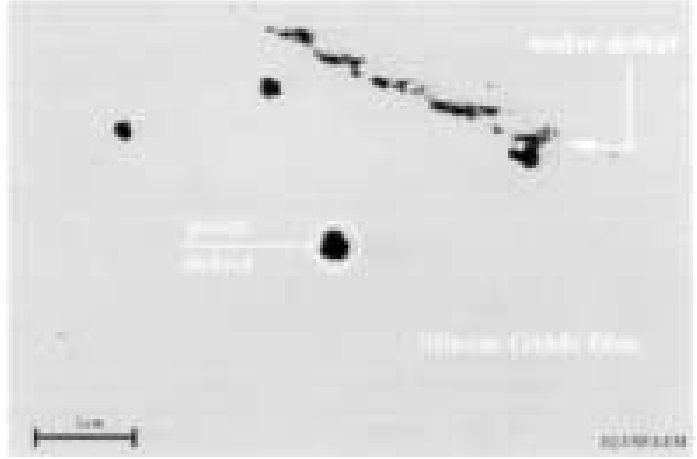

Figure 5. $\mu$-RAMAN image of silicon oxide layer deposited with an argon flow of $135 \mathrm{sccm}$. The surface appears uniform and smooth excepted at wafer defects. These punctual defects appear correlated to the effects observed by AFM.

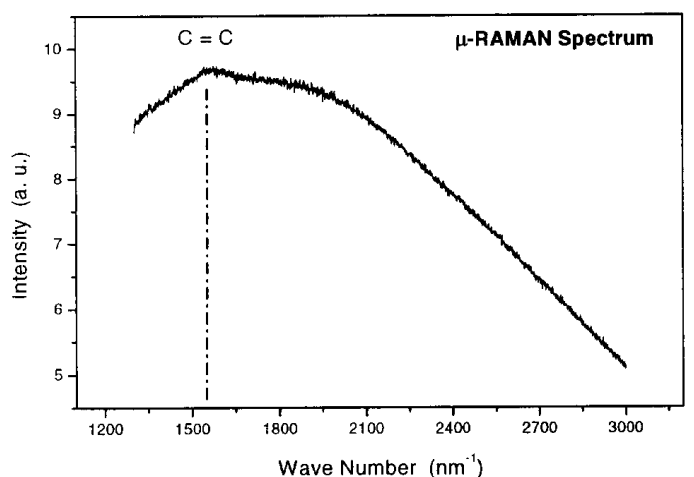

Figure 6. Typical $\mu$-RAMAN Spectrum of a punctual defect as shown Fig. 5. The carbon bonds confirm the presence of unreacted TEOS radicals.

\section{2) Electrical Characterization}

Fig. 7 shows typical J-E curves obtained from the MOS capacitors implemented with TEOS silicon oxide.

Table 3 shows the extracted parameters from the J-E curves for several argon flows. The leakage current density $\left(J_{L K}\right)$ values were obtained with a $4 \mathrm{MV} / \mathrm{cm}$ electrical field applied to the capacitors.

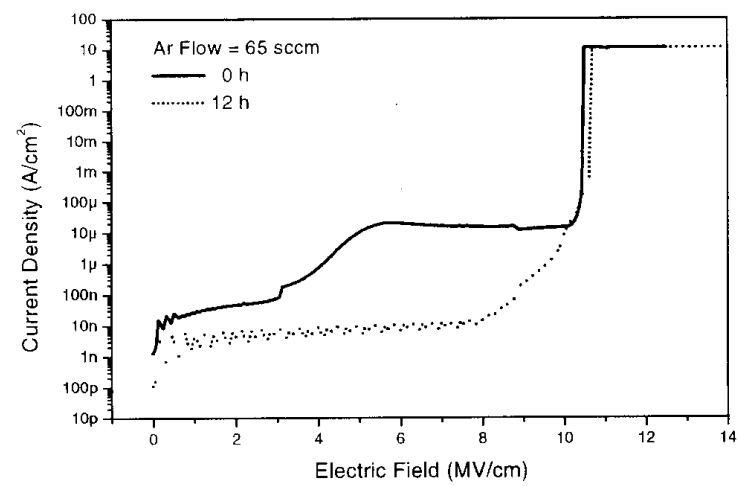

Figure 7. J-E curves from MOS capacitors implemented with an argon flow of $65 \mathrm{sccm}$. The annealing effect decreases the leakage current density and increases the breakdown strength. 
Table 3: Leakage current and breakdown strength according the annealing time and as a function of the argon flow extracted from J-E curves of Fig. 7.

\begin{tabular}{cccc}
\hline \hline $\begin{array}{c}\mathrm{F}_{A r} \\
(\mathrm{sccm})\end{array}$ & $\begin{array}{c}\text { Annealing } \\
\text { Time }(\mathrm{h}):\end{array}$ & $\begin{array}{c}\mathrm{J}_{L K}(\mathrm{~A}) \text { at } \\
4 \mathrm{MV} / \mathrm{cm}\end{array}$ & $\begin{array}{c}\mathrm{E}_{B D} \\
(\mathrm{MV} / \mathrm{cm})\end{array}$ \\
\hline 65 & as-deposited & $7.4 \times 10^{-7}$ & 10.4 \\
& 12 & $5.5 \times 10^{-9}$ & 10.6 \\
150 & as-deposited & $4.4 \times 10^{-6}$ & 9.3 \\
& 12 & $4.4 \times 10^{-9}$ & 10.5 \\
180 & as-deposited & $1.3 \times 10^{-5}$ & 8.5 \\
& $121.5 \times 10^{-8}$ & 10.4 & \\
\hline \hline
\end{tabular}

We can observe an increase of the leakage current density with the argon flow. For the as-deposited samples one can observe the same behavior after annealing. The annealing effectively decreases the leakage current density and increases the breakdown strength. Thus, the best argon flow ranges around $65 \mathrm{sccm}$.

\section{Conclusions}

In order to improve the structural and electrical quality of silicon oxide deposited by PECVD technique and by using TEOS as silicon source, we have introduced argon in the plasma. We have studied silicon oxide films deposited on monocrystalline silicon substrate. The influence of the presence of argon flow in the reactor has different aspects. On one hand, whatever the argon flow, the refractive index, measured by ellipsometry, is very close to thermal oxide one that means that the silicon oxide is always close to stoichiometric composition. On the other hand, an increase of the argon flow decreases first the deposition rate and then increases it. In the same time, the roughness of the silicon surface, analyzed from AFM is continuously increased due to the bombardment of additional species present in the plasma. Some punctual defects were also detected but they appear at the structural defects of the substrate surface. These carbon-based defects are more present when the deposition rate increases as detected by AFM analysis because they can be rapidly overlayed.

As a conclusion, by the argon flow it is possible to get a silicon oxide film with high quality in term of refractive index, smoothness, and uniformity. The silicon substrate surface has to be free of defects to minimize punctual defects in the silicon oxide film, which could affect the electrical behavior. The best results, are for the films with the lowest etching rate, which means denser film, are obtained for an argon flow that ranges from 65 to $80 \mathrm{sccm}$, that corresponds also to the lowest leakage current density.

\section{Acknowledgements}

The authors are grateful to Dr. Mônica A. Cotta, N. M. Hassan and Dr. Sebastião S. G. S. Filho for the AFM analysis, to Dr. M. L. P. Silva for the $\mu$-RAMAN measurements, to Dr. R. D. Mansano and Dr. L. S. Zambon for their help at the cleaning room, to Carlos C. Nascimento and Gilberto K. Nishioka for their technical support. Financial support from FAPESP, $\mathrm{CNPq}$ CAPES/COFECUB and FINEP are gratefully acknowledged.

\section{References}

[1] F. Fracassi R. d'Agostino, and P Favia J. Electrochem, Soc., 139, 2636 (1992).

[2] S.C. Deschmukh and E.S. Aydil, J. Vac. Sc. Technol. B 14738 (1996).

[3] K. Mourgues, F. Raoult, T. Mohammed-Brahim, D. Briand, O. Bonnaud, Mat. Res. Soc. Symp. Proc., 471, 155 (1997).

[4] C. E. Viana, A. N. R. da Silva, and N. I. Morimoto, "Argon Influence in the PETEOS Silicon Oxide Deposition Process", Proc. of International Conference on Microelectronics and Packaging, Curitiba-PR (Brazil), 1998, pp. $481-483$

[5] N. I. Morimoto, J. W. Swart, "A Cluster Tool System for Silicon Oxide Deposition"; Proc. of X Congresso da Sociedade Brasileira de Microeletrônica, Canela-RS (Brazil), july 1995, pp. 393-409

[6] N. I. Morimoto, J. W. Swart "Development of a Cluster Tool and Analysis of Deposition of Silicon oxide by TEOS $/ \mathrm{O}_{2}$ PECVD", Rapid Thermal and Integrated Processing V, Mat. Res. Soc. Symp. Proceedings, vol. 429, 1996, pp. 263-268.

[7] Y. Inoue, O. Tokai "Spectroscopic Studies on Preparation Plasma Sources Sci. Technol., 5, 339 (1996).

[8] R. Etemadi, C. Godet, and J. Perrin, Plasma Sources Sci. Technol. 6, 323 (1997).

[9] P. Lange, L. Schmidt, M. Pelka, P. Hemicker, H. Bernt and Windbracke, J. Electrochem. Soc., 139, 1420 (1992).

[10] H. J. Schliwinski, et al. J. Electrochem. Soc., 139, 1730 (1992).

[11] S. K. Ray, C.K. Maiti, S.K. Lahiri, N.B. Chafrabarti, J. Vac. Sci. Technol. B 10, 1139 (1992). 